\title{
Classificação de sítio em plantio florestal de eucalipto no estado do Amapá
}

\section{Classification of site in eucalyptus forest plantation in the state of Amapá}

\author{
Anthoinny Vittória dos Santos Silva ${ }^{1}$, Rodrigo Galvão Teixeira de Souza², Gabriel Victor \\ Caetano Carvalho Liarte ${ }^{3}$, Robson Borges de Lima ${ }^{4}$, Jadson Coelho de Abreu \\ ${ }^{7}$ Acadêmica de Engenharia Florestal. Universidade do Estado do Amapá - UEAP. Macapá-AP Brasil. E-mail: vittoria.sntsilva@gmail.com \\ *Autor para correspondência \\ ${ }^{2}$ Acadêmico de Engenharia Florestal. Universidade do Estado do Amapá - UEAP. Macapá Brasil. E-mail: rodrigosouza55@hotmail.com \\ ${ }^{3}$ Acadêmico de Engenharia Florestal. Universidade do Estado do Amapá - UEAP. Macapá Brasil. E-mail: gv326766@gmail.com \\ ${ }^{4}$ Doutor em Ciências Florestais. Docente da Universidade do Estado do Amapá-UEAP. Macapá-AP Brasil. E-mail: rbl_florestal@yahoo.com.br \\ ${ }^{5}$ Doutor em Ciência Florestal. Docente da Universidade do Estado do Amapá - UEAP. Macapá-AP Brasil. E-mail: \\ jadsoncoelhoabreu@hotmail.com
}

\section{Palavras-chave}

Crescimento e produção

Ajuste de equação

Mitscherlich

\begin{abstract}
A economia do setor florestal brasileiro é principalmente movida pelo plantio de eucalipto, devido suas características de crescimento rápido e diversidade em relação ao uso. Sendo um produto florestal importante, é essencial que o manejador de florestas plantadas tenha conhecimento de variáveis importantes para tomadas de decisões, como a prognose. Através das estimativas se pode classificar sítios florestais, por meio do método direto que relaciona a altura dominante (Hdom) e a idade (I). A curva-guia é um dos métodos mais utilizados para a construção das curvas de índice de local, portanto, o objetivo do presente estudo foi efetuar a classificação de sítio em plantio florestal de eucalipto no estado do Amapá usando esse método direto. Foram avaliados os modelos estatísticos de Schumacher, Chapman-Richards, Mitscherlich e Logístico 3 parâmetros. E como critério de avaliação foram utilizados: coeficiente de determinação $\left(R^{2}\right.$ ajs) e a raiz quadrada do erro médio (RQEM). E para a geração das curvas de índice de sítio, adotou-se como idade de referência de 60 meses para todas as equações ajustadas. Todas as análises foram efetuadas no software R. As curvas foram construídas para todos os modelos e constatou-se que o que obteve melhor desempenho foi o modelo de Mitscherlich.
\end{abstract}

\section{Keywords}

Growth and production Equation adjustment Mitscherlich

\begin{abstract}
The economy of the Brazilian forestry sector is mainly driven by the planting of eucalyptus, due to its characteristics of rapid growth and diversity in relation to use. Being an important forest product, it is essential that the planted forest manager has knowledge of important variables for decision making, such as prognosis. Through the estimates it is possible to classify forest sites, using the direct method that relates the dominant height (Hdom) and age (I). The guide curve is one of the most used methods for the construction of the site index curves, therefore, the objective of the present study was to classify the site in eucalyptus forest plantation in the state of Amapá using this direct method. The statistical models of Schumacher, Chapman-Richards, Mitscherlich and Logistic 3 parameters were evaluated. And as evaluation criteria were used: determination coefficient ( $\left.R^{2} a j s\right)$ and root-mean-square error (RMSE). And for the generation of the site index curves, the reference age of 60 months was adopted for all adjusted equations. All analyzes were performed using the $R$ software. The curves were built for all models and it was found that the one with the best performance was the Mitscherlich model.
\end{abstract}


conforme Pissinin e Schneider (2017) podem ser divididos em três categorias distintas de avaliação: por meio da vegetação indicadora; através dos fatores relativos ao clima, solo e a fisiografia; e a avaliação mais utilizada, denominada de índice de local ou sítio, que é dada pela relação da variável dependente altura dominante (Hdom) e independente idade (I).

Por meio dos índices de sítio, pode-se avaliar a qualidade do local de modo mais sólido, sendo um método quantitativo feito a partir de equações, que possui praticidade e demonstra a interatividade dos fatores naturais no crescimento em altura (CAMPOS; LEITE, 2017). Há diversos métodos de aplicabilidade desse método direto, como método de atribuição preliminar de índices de local, método da curva-guia, método da equação das diferenças, método de Hammer e Método da predição de parâmetros. Todavia, um dos mais empregados é o método da curva-guia, possuindo uma melhor aplicabilidade com resultados satisfatórios para florestas plantadas de eucalipto (MIGUEL et al., 2011; PEGO; ASSIS; CABACINHA, 2015; RETSLAFF et al., 2015; SILVA et al., 2017).

Diante do exposto, o objetivo do presente trabalho foi classificar a capacidade de sítios de povoamento de eucalipto no estado do Amapá, empregando modelos matemáticos utilizando método da curva-guia.

\section{MATERIAL E MÉTODOS}

Os dados foram obtidos de um plantio florestal comercial, onde a área de estudo está localizada entre os hortos florestais denominados de: Araguari, Asa branca, Bacuri, Capivara, Flexal, Matapi, Pedreira e Peixe-Boi, envolvendo os municípios de Ferreira Gomes (Latitude: 0.85376, Longitude: -51.1941/ 0 51' 14" Norte, 51 11' 39" Oeste-Altitude de 4m - Classificação climática de Köppen-Geiger: Am) e Macapá
(Latitude: 0.704386, Longitude: -51.4096 / 0 42' 16" Norte, $51^{\circ} 24^{\prime} 35^{\prime \prime}$ Oeste - Altitude de $35 \mathrm{~m}$ - Classificação climática de Köppen-Geiger: Am). A classificação oficial do clima do Amapá é "tropical superúmido".

A construção das curvas de índice de local para avaliação da capacidade de produção do sítio foi efetuada pelo método direto que é dado pela relação entre a altura dominante (Hdom) e idade (I). Utilizou-se um dos métodos alternativos a curva-guia, e, foram avaliados os ajustes do modelo estatístico intrinsecamente linear de Schumacher e não lineares de Chapman-Richards, Mitscherlich e Logístico 3 parâmetros. (Tabela 1).

Os ajustes das equações foram efetuados por meio do Método de Máxima Verossimilhança. Adotou-se o nível de significância de $5 \%$ de probabilidade para os coeficientes ( $p$ valor $<0,5)$. E como critério de avaliação foram utilizados: coeficiente de determinação ( $R^{2}$ ajs) e a raiz quadrada do erro médio (RQEM). E para a geração das curvas de índice de sitio, adotou-se como idade de referência de 60 meses para todas as equações ajustadas. Todas as análises foram efetuadas no software R (R DEVELOPMENT CORE TEAM, 2014).

$$
\begin{gathered}
R^{2} \text { ajs }=\left(1-\left(\frac{\text { SQres }}{S Q t}\right) *\left(\frac{n-1}{n-p}\right)\right) * 100 \\
R Q E M=\sqrt{\frac{\sum_{i=1}^{N}(x i-\hat{x} i)^{2}}{N}}
\end{gathered}
$$

Em que: $\mathbf{x i}$ = valor observado da variável dependente; $\hat{\varkappa} \mathbf{i}$ = valor estimado da variável dependente; $\mathrm{N}=$ número de dados observados; $\mathrm{p}=$ número de coeficientes do modelo; SQres = soma dos quadrados dos resíduos; SQt = soma dos quadrados total.

Tabela 1. Modelos utilizados para as estimavas da altura dominante (Hdom) e classificação da capacidade produtiva para os povoamentos de eucalipto.

\begin{tabular}{c|c|c}
\hline Autor & Modelo Matemático & Modelo Guia \\
\hline Schumacher (1) & $\mathrm{Ln}\left(\mathrm{H}_{\mathrm{dom})}=\beta 0+\beta 1\left(\frac{1}{\text { Ii }}\right)+\varepsilon \mathrm{i}\right.$ & $\operatorname{Ln}\left(\mathrm{H}_{\mathrm{dom})}=\mathrm{Ln}(S) *+\beta 1\left[\left(\frac{1}{\text { Ii }}\right)-\left(\frac{1}{\text { Iref }}\right)\right]\right.$ \\
\hline Chapman-Richards (2) & $\mathrm{H}_{\mathrm{dom}}=\beta_{0} *\left(1-\mathrm{e}^{\left(\beta_{1} * I_{i}\right)}\right)^{\beta_{2}}+\varepsilon_{i}$ & $\mathrm{H}_{\mathrm{dom}}=\mathrm{S} *\left(\frac{\left(1-\mathrm{e}^{\beta 1 * \text { Iref }}\right)}{\left(1-e^{\beta 1 * I i}\right)}\right) \beta 2$ \\
\hline Mitscherlich (3) & $\mathrm{H}_{\mathrm{dom}}=\beta 0-\beta 1 * \beta 2^{I i}+\varepsilon_{\mathrm{i}}$ & $\mathrm{H}_{\mathrm{dom}}=\mathrm{S}-\beta 1 *\left(\beta 2^{\text {Ii }}-\beta 2^{\text {Iref }}\right)$ \\
\hline Logístico 3 parâmetros (4) & $\mathrm{H}_{\mathrm{dom}}=\frac{\beta_{0}}{\left(1+e^{\frac{\left(\beta_{1}+I_{i}\right)}{\beta_{2}}}\right)}+\varepsilon_{\mathrm{i}}$ & $\mathrm{H}_{\mathrm{dom}}=S * \frac{1+e^{\left(\frac{\beta 1+\text { Iref }}{\beta 2}\right)}}{1+e^{\left(\frac{\beta 1+I i}{\beta 2}\right)}}$ \\
\hline
\end{tabular}

$\mathrm{H}_{\text {dom }}=$ altura dominante $(\mathrm{m}) ; \mathrm{S}=$ índice de sítio na idade de referência $(\mathrm{m}) ; \mathrm{I}_{\text {ref }}=$ idade de referência ou idade índice $(60$ meses $) ; \mathrm{I}_{i}=$ idade (60 meses) da parcela no momento da medição da altura; $\beta \mathrm{i}=$ coeficientes dos modelos a serem estimados; $\varepsilon_{\mathrm{i}}=$ erro aleatório 


\section{RESULTADOS E DISCUSSÃO}

Observa-se na tabela 2 os coeficientes e as estatísticas de avaliação dos modelos. Em que o modelo de Logístico e Chapman-Richards apresentaram os melhores valores, seguidamente de Mitscherlich e Schumacher. Todos os modelos testados apresentaram uma satisfatória distribuição residual tendendo a homogeneidade, apesar de apresentar uniformidade em torno da linha média, observa-se uma dispersão mais intensa nas idades superiores, comportamento semelhante demostrado por ZIECH et al. (2016) na construção das curvas de índice de local para Teca (Figura 1).

Contruiu-se as dez curvas de índice de local para cada modelo testado, com uma amplitude de $2 \mathrm{~m}$, com uma variação de $10 \mathrm{~m}$ a $28 \mathrm{~m}$ na idade de referência de 60 meses (Figura 2). Todos os modelos geraram curvas anamórficas como observado na figura 2. Em contrapartida a esse estudo, segundo Melo et al. (2017) os modelos que apresentam o menor erro que são eficientes para a construção das curvas de indice de local, partindo desse princípio, o que deveria apresentar melhor desempenho seria o Logístico (tabela 2), todavia, o comportamento das curvas não englobaram os dados das idades iniciais. No estudo apresentado por Silva (2017) essa regressão também apresentou o não adequamento aos dados. $O$ de Schumacher além de valores maiores de RQEM (Tabela 2), provavelmente este comportamento é pelo modelo está na sua forma linearizada, Pego et al. (2016) apresentou em seu estudo de classificar os sítios em povoamentos de eucalipto em uma microrregião de Minas Gerais, um baixo desempenho nas estimativas do modelo linearizado de Schumacher, não fazendo uso para classificação. Na figura 2, se observa que as curvas construídas apresentaram comportamento similiar da equação anterior.

O Modelo de Chapman-Richards não englobou os dados nas primeiras idades e na classe 10 ficou sem observações. Em contrapartida, na literatura em geral o modelo que apresenta melhor desempenho é o Chapman-Richards, como demostrado no estudo de Restlaff et al. (2015), que fez aplicação para o Eucalyptus grandis na região do Campos Gerais, em que o autor afirma que o modelo apresenta características que favorecem a construção das curvas. Também na região do Paraná, o autor Santana (2008) utilizou o mesmo para a avaliação da capacidade produtiva do local. Cruz et al. (2008) demonstrou o desempenho da equação ajustada escolhida para descrição das tendências de crescimento de Teca. Lima filho et al. (2012) estudou a regressão em diferentes distribuições simétricas dos erros para modelar o crescimento de clones de eucalipto, demonstrando sua relevância. Santos et al. (2018) utilizou-o para classificação de sítio através do método da diferença algébrica comparando a regressão quantílica (RQ), porém não apresentou resultados superiores a $\mathrm{RQ}$.

Mitscherlich foi o modelo testado que melhor demonstrou as tendências da altura dominante, englobando todos os dados (Figura 2). A explicação do desempenho satisfatório, é por ser uma regressão não linear, caracterizando-a em ser parcimoniosa, possuindo confiabilidade nas estimativas e facilidade na elucidação dos parâmetros (assíntota, ponto de inflexão e escala) (CALEGARIO et al., 2005; SILVA, 2017). Em contraponto, o estudo de Restlaff et al. (2015) demostrou baixo desempenho do modelo, assim como o trabalho de Chaves et al. (2016), no entanto para essa base dados possuiu

Tabela 2. Coeficientes e estatísticas ( $\mathrm{R}^{2}$ ajs-Coeficiente de determinação e RQEM-Raiz quadrada do erro médio) dos modelos testados para construção das curvas de sítio.

\begin{tabular}{|c|c|c|c|c|}
\hline Autor & Modelo Matemático & Coeficientes & $\mathrm{R}^{2} \mathrm{ajs}$ & RQEM \\
\hline Schumacher (1) & $\operatorname{Ln}\left(\mathrm{H}_{\mathrm{dom})}=\beta 0+\beta 1\left(\frac{1}{I i}\right)+\varepsilon \mathrm{i}\right.$ & $\begin{array}{c}\beta 0=3,4785 \\
\beta 1=-25,1653\end{array}$ & 0,6464 & 14,91 \\
\hline $\begin{array}{l}\text { Chapman- } \\
\text { Richards (2) }\end{array}$ & $\mathrm{H}_{\mathrm{dom}}=\beta 0 *\left(1-\mathrm{e}^{\beta 1 * \mathrm{Ii}}\right)^{\beta 2}+\varepsilon \mathrm{i}$ & $\begin{array}{c}\beta 0=25,6373 \\
\beta 1=0,3377 \\
\beta 2=1,5364\end{array}$ & 0,6473 & 2,7379 \\
\hline Mitscherlich (3) & $\mathrm{H}_{\mathrm{dom}}=\beta 0-\beta 1 * \beta 2^{I i}+\varepsilon_{\mathrm{i}}$ & $\begin{array}{c}\beta 0=26,5291 \\
\beta 1=31,6675 \\
\beta 2=0,9691\end{array}$ & 0,6470 & 2,7390 \\
\hline $\begin{array}{c}\text { Logístico } 3 \\
\text { parâmetros (4) }\end{array}$ & $\mathrm{H}_{\mathrm{dom}}=\frac{\beta_{0}}{\left(1+e^{\frac{\left(\beta_{1}+I_{i}\right)}{\beta_{2}}}\right)}+\varepsilon_{\mathrm{i}}$ & $\begin{array}{c}\beta 0=23,7002 \\
\beta 1=5,1463 \\
\beta 2=0,0665\end{array}$ & 0,6483 & 2,7341 \\
\hline
\end{tabular}

$\mathrm{H}_{\mathrm{dom}}=$ altura dominante $(\mathrm{m}) ; \mathrm{S}=$ índice de sítio na idade de referência $(\mathrm{m}) ; \mathrm{I}_{\text {ref }}=$ idade de referência ou idade índice $(60$ meses); $\mathrm{I}_{i}=$ idade (60 meses) da parcela no momento da medição da altura; $\beta \mathrm{i}=$ coeficientes dos modelos a serem estimados; $\varepsilon_{\mathrm{i}}=$ erro aleatório 
Figura 1. Distribuição residual das equações ajustadas.
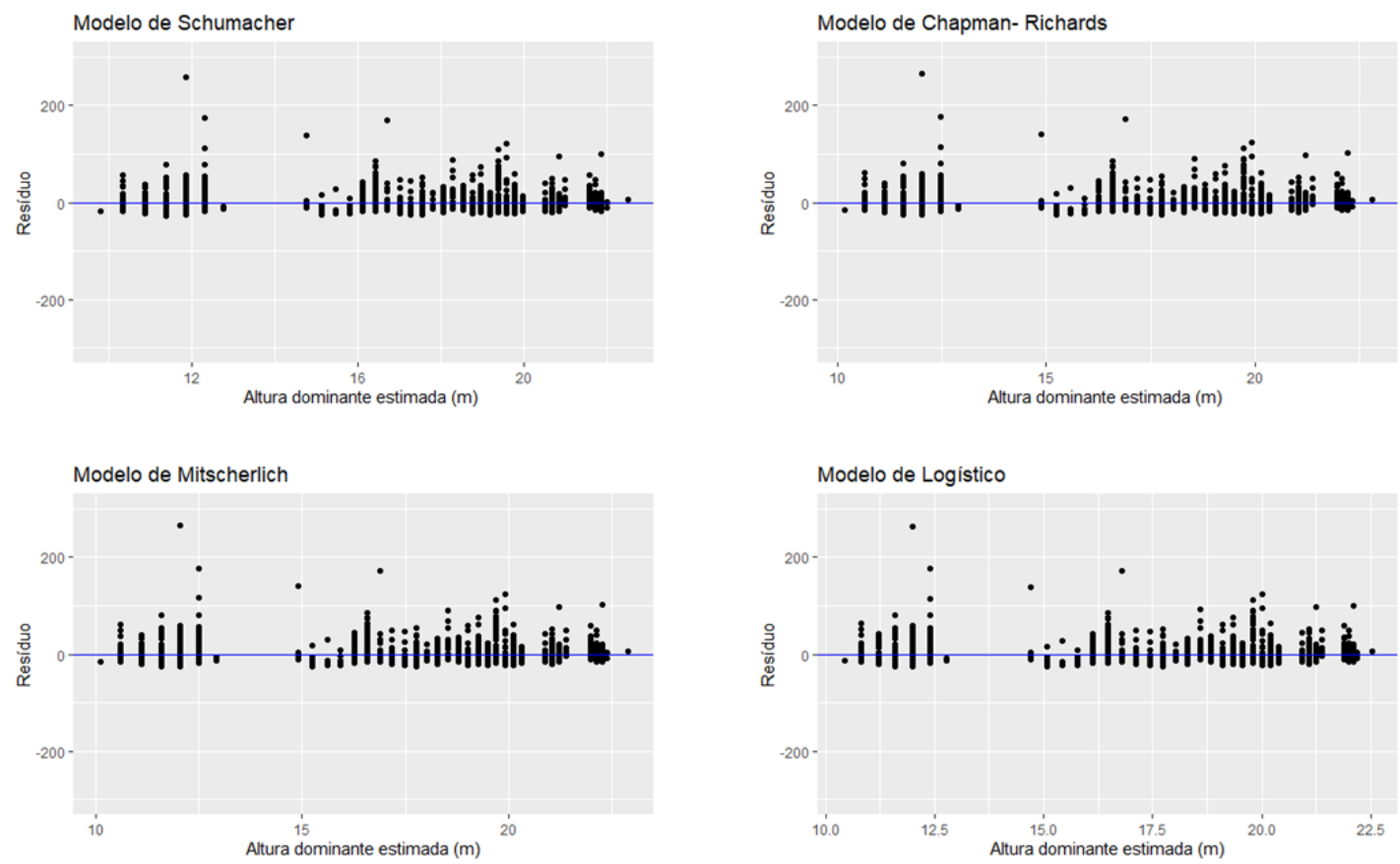

Figura 2. Curvas de índice de sítio pra todos os modelos testados.
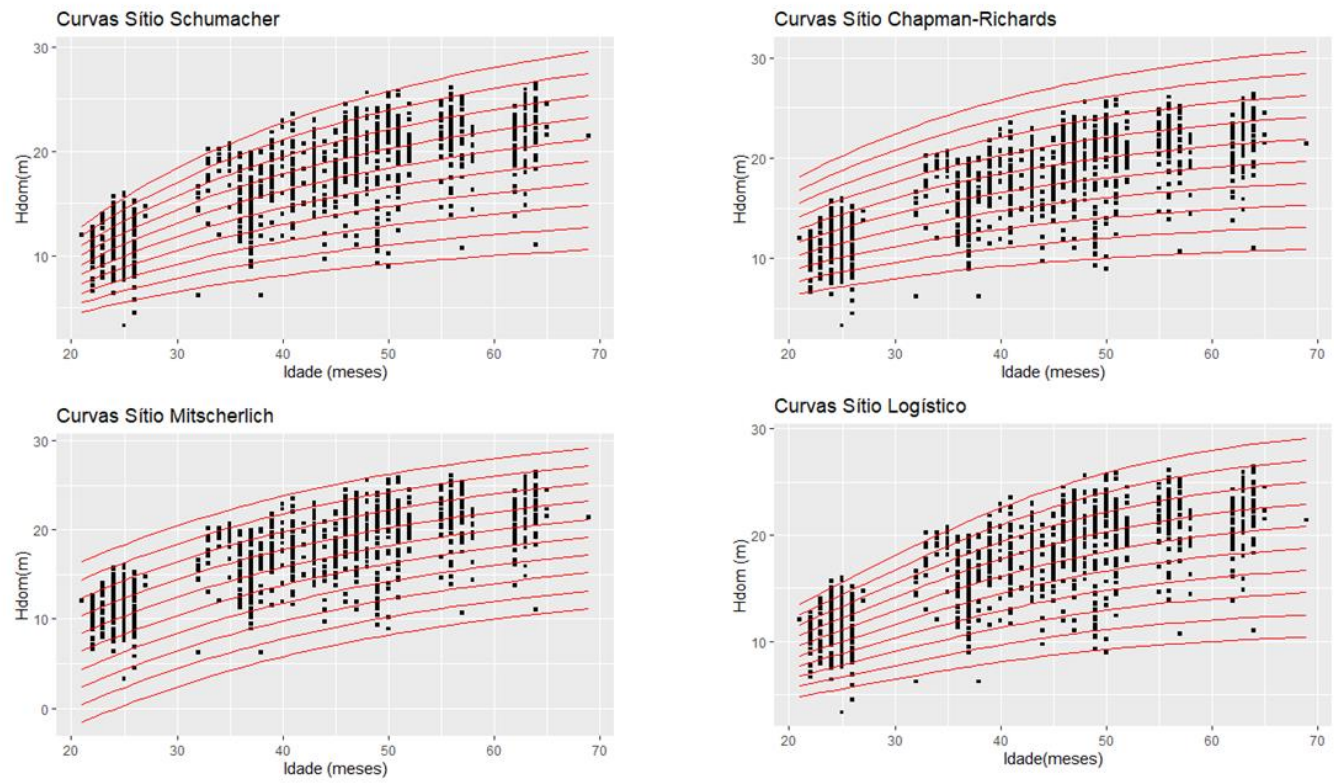

o melhor desempenho para a construção das curvas de índice de sítio.

\section{CONSIDERAÇÕES FINAIS}

Todos os modelos apresentaram coeficientes com ajustes satisfatórios, boas estatísticas e distribuição gráfica residual. Porém, o melhor modelo testado para a construção das

curvas de índice de sítio foi o modelo de Mitscherlich, uma regressão não linear que possui característica que auxiliam no comportamento sigmoide das curvas.

\section{AGRADECIMENTOS}

À Universidade do Estado do Amapá (UEAP), ao laboratório de Manejo Florestal e ao grupo de pesquisa 
Métodos quantitativos aplicados aos Recursos florestais pelo apoio para o desenvolvimento desse estudo.

\section{REFERÊNCIAS}

CALEGARIO, N.; DANIELS, R. F.; MAESTRI, R.; NEIVA, R. Modeling dominant height growth based on nonlinear mixed-effects model: a clonal Eucalyptus plantation case study, Forest Ecology and Management, Amsterdam, v. 204, n. 1, p. 11-20, 2005.

CAMPOS, J. C. C.; LEITE, H. G. Mensuração florestal: perguntas e respostas. 3. Ed. Viçosa: Editora UFV, 2017. 291 p.

CARVALHO, S.P.C.; CALEGARIO, N.; SILVA, F.F.; BORGES, L.A.C.; MENDONÇA, A.R. LIMA, M.P. Modelos não lineares generalizados aplicados na predição da área basal e volume de Eucalyptus clonal. Cerne, v. 17, n. 4, p. 541-548, 2011.

CHAVES, A.G.S.; DRESCHER, R.; CALDEIRA, S.F.; MARTINEZ, D.T.; VENDRUSCOLO, D.G.S. Capacidade produtiva de Tectona grandis L.f no Sudoeste de Mato Grosso. Sci. For., Piracicaba, v. 44, n. 110, p. 415-424, jun. 2016.

COSENZA, D.N.; LEITE, H.G.; MARCATTI, G.E.; BINOTI, H.B.; ALCÂNTARA, A.E.M.; RODE, R. Classificação da capacidade produtiva de sítios florestais utilizando máquina de vetor de suporte e rede neural artificial. Sci. For., Piracicaba, v. 43, n. 108, p. 955-963, dez. 2015.

CRUZ, J. P.; LEITE, H. G.; SOARES, C. P. B.; CAMPOS, J. C. C.; SMIT, L. NOGUEIRA, G. S. Curvas de crescimento e de índice de local para povoamentos de Tectona grandis em Tangará da Serra, Mato Grosso. Revista Árvore, Viçosa, v. 32, n. 4, p. 679-685, 2008.

EMBRAPA - Empresa Brasileira de Pesquisa Agropecuária. Plantações florestais: Geração de benefícios com baixoimpacto ambiental. 2016. Disponível em: <http://iba.org/images/shared/Biblioteca/Estudo_embrapa. pdf> Acesso em: 01 de Março de 2020.

GONÇALVES, J.C. CARVALHO, S.P.C.; OLIVEIRA, A.D.; GOMIDE, L.R. Comparação dos modelos prognósticos de Clutter e da função logística. Pesq. flor. bras., Colombo, v. 36, n. 87, p. 311-317, jul.-set. 2016.

INDÚSTRIA BRASILEIRA DE ÁRVORES. Relatório IBÁ 2019. Brasília: IBÁ, 2019. Disponível em: < https://iba.org/datafiles/publicacoes/relatorios/ibarelatorioanual2019.pdf> . Acesso em: 01 de março de 2020.

LIMA FILHO, L. M. A.; SILVA, J. A. A.; CORDEIRO, G. M.; FERREIRA, R. L. C. Modelagem do crescimento de clones de Eucalyptus usando o modelo de Chapman-Richards com diferentes distribuições simétricas dos erros. Revista Ciência Florestal, Santa Maria, v. 22, p. 777 - 785, 2012.
MELO, E.A.; CALEGARIO, N.; MENDOÇA, A.R.; POSSATO, E.L. ALVES, J.A.; JÚNIOR ISAAC, M.A. Modelagem não linear da relação hipsométrica e do crescimento das árvores dominantes e codominantes de Eucalyptus sp. Ciência Florestal, Santa Maria, v. 27, n. 4, p. 1325-1338, out.-dez., 2017.

MIGUEL, E. P.; ENCINAS, J. I.; REZENDE, A.V.; FERREIRA, J. C. S.; AGUIAR, G.P. Classificação de sítio para plantios de Eucalyptus urophylla em Niquelândia, estado de Goiás. Enciclopédia Biosfera, Goiânia, v. 7, n. 12, p. 1- 11, mai. 2011.

PEGO, M. F. F.; ASSIS, A. L. de; CABACINHA, C. D. Classificação de sítios florestais em povoamentos de eucalipto na microrregião de Salinas, Minas Gerais. Enciclopédia Biosfera, Goiânia, v. 11, n. 1, p. 1-5, jun. 2015.

PISSININ, L. Z.; SCHNEIDER, P. R. Tendências de crescimento em altura dominante de dois clones de Eucalyptus saligna Smith implantados em distintas condições de solo. Ciênc. Florest., Santa Maria, v. 27, n. 1, p. 263-275, mar. 2017.

R CORE TEAM R: A language and environment for statistical computing $\mathrm{R}$ foundation for Statistical Computing. Vienna, 2014. Disponível em: <http://www.R-project.org/>. Acesso em: 20 de fevereiro de 2020.

RETSLAFF, F. A. de S.; FIGUEIREDO FILHO, A.; DIAS, A.N.; BERNETT, L.G.; FIGURA, M.A. Curvas de sítio e relações hipsométricas para Eucalyptus grandisna região dos campos gerais. Paraná, Cerne, Lavras, v. 21, n. 2, p. 219225, jan. 2015.

SANTANA, C. J. O. Simulação do crescimento e da produção em plantações desbastadas de Eucalyptus grandis com diferentes procedimentos de obtenção dos parâmetros da distribuição Weibull. 2008. 100 p. Dissertação (Mestrado em Engenharia Florestal) - Universidade Federal do Paraná, Curitiba, 2008.

SANTOS, C.J.; THIERSCH, C.R.; MOREIRA, M.F.B. Regressão não linear quantílica para classificação de sítio em povoamentos de Eucalyptus sp. Sci. For., Piracicaba, v. 46, n. 120, p. 551-561, dez. 2018.

SILVA, G.C.C. Modelagem do crescimento e da produção florestal em povoamentos de eucalipto desbastado e não desbastado, 2017. Dissertação (mestrado acadêmico) Universidade Federal de Lavras, 2017.

ZIECH, B.G.; SILVA, V.S.M.; DRESCHER, R.; VENDRUSCOLO, D.G.S. Modelos de crescimento em altura dominante e índice de sítio para teca em Glória D’Oeste-MT. Rev. Bras. Biom., Lavras, v.34, n.4, p.533-542, 2016.

Submissão: 07/03/2020

Aprovado para publicação: 14/03/2020 